Archives

\title{
Le Play, ses continuateurs et la question du territoire (1855-1914)
}

\section{Antoine Savoye}

\section{(2) OpenEdition}

\section{Journals}

Édition électronique

URL : http://journals.openedition.org/ccrh/2687

DOI : $10.4000 /$ ccrh.2687

ISSN : 1760-7906

Éditeur

Centre de recherches historiques - EHESS

Édition imprimée

Date de publication : 14 octobre 1995

ISSN : 0990-9141

Référence électronique

Antoine Savoye, «Le Play, ses continuateurs et la question du territoire (1855-1914) », Les Cahiers du Centre de Recherches Historiques [En ligne], 14-15| 1995, mis en ligne le 27 février 2009, consulté le 02 mai 2019. URL : http://journals.openedition.org/ccrh/2687 ; DOI : 10.4000/ccrh.2687

Ce document a été généré automatiquement le 2 mai 2019.

Article L.111-1 du Code de la propriété intellectuelle. 


\title{
Le Play, ses continuateurs et la question du territoire (1855-1914)
}

\author{
Antoine Savoye
}

1 Répondant à la suggestion des fondateurs de notre groupe de travail (cf. le «projet à quatre ans ", mai 1994), j'ai exploré comment la science sociale leplaysienne traite de la question du Territoire ${ }^{1}$. Plus exactement, il s'agit d'une vue cavalière, étant donné l'amplitude de la période considérée ${ }^{2}$ et l'importance numérique des travaux publiés durant cette soixantaine d'années par Le Play et ses continuateurs.

2 La première partie de l'exposé traite de la prise en compte du territoire par la science sociale leplaysienne c'est-à-dire comment celle-ci analyse le rapport du « social » à son « territoire », à travers le concept de Lieu.

3 La deuxième partie concerne un aspect particulier de l'intérêt porté par les leplaysiens aux questions de territoire. Il s'agit de leurs nombreuses recherches sur l'organisation administrative et politique (en France et à l'étranger) considérée sous l'angle territorial.

4 Enfin, la troisième partie évoque les applications pratiques que les leplaysiens - toujours soucieux de coupler science et action, démarche cognitive et engagement social - ont tiré de leur analyse du rapport société/territoire.

\section{Le lieu, une conceptualisation du rapport au territoire}

5 Le principal outil théorique et méthodologique de la science sociale leplaysienne pour l'analyse de l'« espace » et du «territoire » dans leur rapport au social, est le concept de « lieu », élaboré par Le Play ${ }^{3}$ puis retravaillé par Tourville.

\section{La conception de Le Play}

6 Le concept de Lieu est mis en oeuvre tout d'abord dans la monographie de famille, base empirique, chez Le Play, de l'étude des sociétés. 
7 Dans une monographie (effectuée selon des règles de méthode strictement codifiées), la description du Lieu où vit la famille répond au principe de «localisation » des faits sociaux. Ceux-ci, selon Le Play, ne peuvent être étudiés indépendamment de leur territoire car, pour l'auteur des Ouvriers européens, il existe des relations étroites entre une organisation sociale et son cadre géographique.

8 Vingt ans après Les Ouvriers européens, Le Play tente de mettre au point un nouvel instrument d'étude qui évite de passer par la description des familles et permet d'accéder directement à une connaissance globale des organisations sociales. Il s'agit de la monographie de société où le terme de territoire apparaît nommément, sans pour autant annuler le concept de lieu.

9 En effet, le «territoire d'une nation »- l'Angleterre, par exemple -, n'est pas homogène. Il est fait de « lieux » présentant des conditions naturelles diversifiées, occupés et habités différemment, lieux que la science sociale permet de préciser. Le Play parle des « lieux du territoire » dont l'étude constitue une " géographie sociale ».

10 Chaque monographie de société débute par un « tableau préliminaire du territoire occupé par la race et des empreintes que celle-ci y a laissées ${ }^{4}$ ». Cet exposé est, en partie, le résultat d'observations in situ, Le Play conseillant aux auteurs de monographies de " préluder à leur entreprise par de longs séjours dans les diverses parties du territoire qu'elle doit embrasser ». Ajoutant qu'« en chaque lieu, ils ont à conquérir [...] la confiance des familles qu'ils se proposent d'observer ${ }^{5}$ »

11 L'analyse de la «constitution sociale » de chaque société étudiée (qui est la finalité de la monographie) se divise en douze livres. Le premier livre-celui qui concerne plus spécialement sur le territoire - a pour titre "les lieux et la population» (il s'intitule également " précis géographique »). Il comprend dix chapitres :

- aperçu général du territoire

- le climat et le régime atmosphérique

- les forêts et les steppes

- les cultures

- les sols divers et les eaux

- les ressources naturelles fournies par le sol, l'air et les eaux

- les obstacles spéciaux opposés par la nature des lieux

- la population et le travail

- les campagnes et les villes

- les voies commerciales.

Au travers de ces différents chapitres, il s'agit de cerner l'«influence de la nature des lieux sur la constitution sociale ${ }^{6} »$.

\section{L'apport de Tourville}

L'étude du Lieu (autrement dit, des interrelations entre le territoire et le social), est reprise et approfondie par un des principaux disciples de Le Play, Henri de Tourville (1842-1907). Celui-ci, en vue de l'étude méthodique des sociétés, forge un nouvel instrument d'analyse qu'il nomme "nomenclature des faits sociaux» (1883). Il y répertorie et y classe, d'une manière qui se veut exhaustive, les faits constitutifs de la vie sociale, depuis la famille jusqu'à l'État, en passant le travail, la religion, le mode d'existence, etc. 

comportant plus de trois cents items), les relations du «social » à son "territoire » apparaissent essentiellement dans les sept « classes de faits » suivantes :

- le Lieu proprement dit,

- le Voisinage,

- la Commune,

- la Cité,

- le Pays,

- la Province,

- l'Expansion de la race (les migrations, notamment la colonisation).

ces faits sociaux (relevant d'une de ces classes) est étudié à la fois en lui-même et pour ses interrelations avec les autres faits appartenant aux autres classes. Demolins, lui aussi disciple de Le Play et collaborateur de Tourville, forgera plus tard, pour désigner ces relations, le concept de « répercussions ».

Dans la classification générale des faits sociaux établie par Tourville, le « lieu » se place en premier. Considéré comme l'ordre de faits le plus simple, il désigne «l'ensemble des phénomènes matériels naturels, réunis sur un point déterminé de l'espace. C'est en un mot le lieu physique ». Il se décompose en cinq rubriques : sol et eaux, sous-sol, air, productions végétales et animales.

Outil de classification, la nomenclature est aussi un guide pour l'observation des sociétés. L'enquête concernant le lieu, dans le cadre de l'étude monographique d'une société donnée, ne consiste pas en de simples constatations géographiques, mais en des énoncés mettant en relation un fait physique avec un fait social. Les élèves de Tourville parlent même de «lieu physique social », ensemble des phénomènes naturels matériels, à l'aide ou à l'encontre desquels l'action sociale a pu se produire ${ }^{7}$.

atrelation entre géographie physique et réalité sociale, développée dans La Science sociale vue du groupe dont Tourville est le mentor scientifique -, à partir de 1886, est partagée par Vidal de la Blache qui, une vingtaine d'années plus tard, parlera d'un " enchaînement, une liaison entre faits géographiques et sociaux, entre le sol, les cultures, les occupations, les groupements, les habitations ».

\section{Une science du rapport société/territoire}

19 L'importance accordée au territoire dans l'étude des sociétés se traduit chez les leplaysiens, par des recherches mettant spécialement l'accent sur le rapport société/ territoire. Il s'agit tout d'abord de recherches comprenant nécessairement une enquête «sur les lieux » et aboutissant à une monographie de famille ou de société telle que Le Play le prônait.

20 Ce modèle se diversifie à partir de Tourville dont la Nomenclature des faits sociaux permet à d'autres travaux de voir le jour, sous forme de monographies de "pays » (cf. Robert Pinot), voire de sociétés plus vastes (cf. Paul de Rousiers).

21 Un peu plus tard, sont aussi entreprises des monographies de communes dont le cadre d'analyse est mis au point et perfectionné par d'autres continuateurs de Le Play comme Cheysson, Guérin ou du Maroussem. 


\section{L'organisation du territoire}

La prise en compte du territoire apparaît donc comme une des caractéristiques essentielles du paradigme de la science sociale leplaysienne, par laquelle cette dernière se distingue de ses rivales telles que la sociologie durkheimienne ou l'organicisme de René Worms.

Il en découle que les questions territoriales se trouvent au centre de nombreux travaux réalisés par les continuateurs de Le Play. On doit mentionner en particulier les études qui, depuis La Réforme sociale en France de Le Play jusqu'à la réflexion régionaliste du début du siècle, portent sur l'organisation administrative et politique territoriale (découpage, instances de gouvernement, attributions, etc.). Leur objet central est la centralisation administrative et politique, souvent étudiée d'un point de vue historique et par des études comparées, notamment pour ses effets sociaux, appréhendés au niveau d'entités telles que la commune. Ensemble, ces travaux constituent une véritable spécialité au sein du corpus leplaysien.

\section{Le territoire, objet d'investissement social et politique}

La conception selon laquelle les faits sociaux ne peuvent être envisagés indépendamment de leur territoire, n'est pas que de nature cognitive. Elle a aussi une portée pratique. Elle amène les leplaysiens "réformateurs sociaux" (qui, dans leurs travaux, allient visée explicative et visée prescriptive), à prôner et à entreprendre des actions sociales «territorialisées». Autrement dit, à agir sur le social à partir de sa concrétisation territoriale (ou de sa "morphologie sociale », comme dira plus tard Halbwachs, à la suite de Durkheim).

\section{Les « autorités sociales » vecteurs de l'action sociale locale}

Le Play et ses continuateurs encouragent l'émergence d' " autorités sociales ", élite locale dont la pratique sociale exemplaire s'exerce dans un lieu concret («atelier de travail», " voisinage ", « commune ", etc.). C'est à cette échelle locale que leur action prend tout son sens et son effet.

\section{Patronage et urbanisme, deux formes d'action localisée}

La prise en compte du lieu par la science sociale débouche en particulier sur deux applications spécifiques :

- premièrement, le Patronage (dans l'industrie comme dans l'agriculture), dont la théorie et les principes d'action sont formulés par Le Play, repris et amplifiés par ses disciples de la Société d'économie sociale

- deuxièmement, sur la base des travaux de Tourville, l'Urbanisme, théorie praxéologique dont Alfred Agache est un des fondateurs. Celui-ci conçoit l'urbanisme comme une intervention raisonnée sur certains éléments morphologiques de la vie sociale, dégagés par la Nomenclature (i.e., la Cité), en vue d'agir sur les autres classes de faits sociaux, notamment le Mode d'existence. 


\section{La décentralisation administrative et politique} que la région (province), instance nouvelle à recréer.

\section{Conclusion} phénomène mériterait d'être étudié de plus près.

\section{Sources} Méridiens Klincksieck, 1989). consacré à la « vie provinciale ", $2^{\mathrm{e}}$ semestre 1904 ; 1994.

La science sociale alimente également la réflexion et l'action sur l'organisation politicoadministrative de la France, en particulier ce qui touche aux collectivités territoriales.

Les leplaysiens militent pour la création d'entités harmonieuses et équilibrées où l'initiative privée, individuelle et collective, pourra se substituer à l'État et à sa bureaucratie dont les tendances centralisatrices doivent être contenues. Cette réorganisation concerne aussi bien la commune que le canton et l'arrondissement, ainsi

Des représentants de la science sociale leplaysienne comme Picot, vice-président de la Commission Ribot en 1895, participent directement à la réforme administrative. Au tournant du siècle, l'action en faveur de la décentralisation tend à laisser la place à un soutien au régionalisme naissant dont l'École de Le Play se fait volontiers le support.

Au terme de cette enquête, menée au sein du vaste corpus leplaysien, forcément incomplète ${ }^{8}$ et qu'il faudrait approfondir, quelques points forts se dégagent.

Ainsi, restituer la genèse du concept de lieu, puis ses avatars (notamment ses rapports avec la notion de milieu), me semble une piste de travail à emprunter pour mieux comprendre avec quels outils les sciences sociales naissantes pensent l'espace et le territoire. De même, la réflexion amorcée par les leplaysiens sur la centralisation et ses effets sociaux pourrait être prise comme un exemple significatif d'objet travaillé puis en grande partie délaissé par la «sociologie», au profit d'autres disciplines. Un tel

Ces deux exemples nous indiquent quel contenu concret pourrait prendre l'histoire des sciences sociales de l'espace et du territoire que nous ambitionnons.

F. Le Play, Les Ouvriers européens, 1855 ; La Réforme sociale en France, 1864 ; L'Organisation du travail, 1870; La Constitution de l'Angleterre, 1875; La Méthode sociale, 1879 (réédition

La Science sociale (1886-1914). Notamment, Henri de Tourville, « La Science sociale est-elle une science? ", janvier-décembre 1886 ; Prosper Prieur, « Le Lieu », juillet 1886 ; Robert Pinot, «Cours de Méthode de la Science sociale », mars 1891-août 1893 ; Daniel Touzaud, « La décentralisation administrative », avril et septembre 1895, septembre 1896

La Réforme sociale (revue créée en 1881). Par exemple, Jules Lacointa, «L'organisation municipale dans divers pays, d'après les travaux de la Société d'économie sociale », $1^{\text {er }}$ juillet et $1^{\mathrm{er}}$ août 1882 ; Jules Angot des Rotours, «La Province et ses institutions 1789-1889 ", $1^{\text {er }}$ janvier 1890 ; les actes du congrès de la Société d'économie sociale

Catherine Bruant, "Alfred Agache, urbaniste et sociologue », Les Etudes sociales, $n^{\circ} 122$, 
J. Charles-Brun, Le Régionalisme, 1911.

Joseph Ferrand, Les Institutions administratives en France et à l'étranger 1879; Pays libres, 1884.

B. Kalaora et A. Savoye, Les Inventeurs oubliés. Le Play et ses continuateurs aux origines des sciences sociales, Champ Vallon, 1989.

Ferdinand Lepelletier, De la décentralisation administrative et des économies à réaliser, 1896.

Pierre du Maroussem, Les Enquêtes. Théorie et pratique, 1900

Paul de Rousiers, La Vie américaine, 1892

Paul Roux, Précis de science sociale, 1914.

Vidal de La Blache, « Les pays de France », La Réforme sociale, $1^{\mathrm{er}}$ septembre 1904.

\section{NOTES}

1. Précisons, dès à présent, que le mot « territoire » est peu employé (et celui d'« espace » encore moins) par les leplaysiens. Il ne fait pas partie de leur terminologie de base, même si les questions sociales sont fréquemment examinées par les leplaysiens sous l'angle territorial.

2. Mon point de départ, 1855 , correspond à la date de publication des Ouvriers européens ; quant à la première guerre mondiale, elle marque, pour l'École de Le Play comme pour beaucoup de courants intellectuels nés au XIX ${ }^{\mathrm{e}}$ siècle, un tournant, après lequel ceux-ci entrent dans une autre période.

3. Pour une présentation générale de Le Play et son école, on peut se reporter à B. Kalaora et A. Savoye, Les Inventeurs oubliés. Le Play et ses continuateurs aux origines des sciences sociales, Champ Vallon, 1989. On trouve également une présentation ramassée dans l'introduction à Le Play, La Méthode sociale, 1879, réédition Méridiens-Klincksieck, 1989.

4. La Constitution de l'Angleterre, p. XX

5. Ibid.

6. Alexis Delaire, «Index alphabétique », La Constitution de l'Angleterre.

7. P. Prieur, La Science sociale, 1886, p. 31.

8. Ainsi, j'ai dégagé les aspects méthodologiques généraux (comment les leplaysiens objectivent le rapport territoire/société), passant sous silence les résultats concrets des recherches effectuées. D'autre part, j'ai souligné les principales lignes de forces de la science sociale leplaysienne, au détriment de l'analyse de ses contradictions internes et de ses contextes d'émergence. 


\section{AUTEUR}

\section{ANTOINE SAVOYE}

Groupe Espace et territoire dans les sciences sociales 